\title{
In Vitro Antibacterial Activity of Different Honey Samples against Clinical Isolates
}

\author{
Mahasin Ahmed Wadi \\ Medical Microbiology, Department of Medical Surgical College of Nursing, Princess Nourah Bint Abdulrahman University, \\ Riyadh, Saudi Arabia \\ Correspondence should be addressed to Mahasin Ahmed Wadi; mawadihassan@pnu.edu.sa
}

Received 31 July 2021; Revised 19 December 2021; Accepted 29 December 2021; Published 21 January 2022

Academic Editor: Khalid Mehmood

Copyright (C) 2022 Mahasin Ahmed Wadi. This is an open access article distributed under the Creative Commons Attribution License, which permits unrestricted use, distribution, and reproduction in any medium, provided the original work is properly cited.

\begin{abstract}
Background. The emergence of multi-drug-resistant organisms has created a lot of clinical problems. Hence, there is a need to find natural alternative treatment to counter the multi-drug-resistant organisms. Honey has a well-established usage as wound dressing in ancient and traditional medicine. Objective. The objective of this study is to establish a baseline for the antibacterial activity of 32 global raw natural and commercial various honey samples against 8 clinical isolates. Methods. Thirty-two honey samples (raw and commercial honey) collected from different global countries with different floral origins were tested in vitro for antibacterial activity against 8 clinical isolates collected from patients, at private hospital from Sudan, using disk diffusion technique. The following 6 epsilometer tests (Etest), amoxicillin, ciprofloxacin, cefotaxime, chloramphenicol, gentamicin, and tetracycline, were used against 8 clinical isolates for Minimum Inhibitory Concentration (MIC). Results. The following 8 clinical isolates were identified by conventional bacteriological methods: Staphylococcus aureus, (S. aureus) Escherichia coli (E. coli), Klebsiella pneumoniae (K. pneumoniae), Pseudomonas aeruginosa (P. aeruginosa), Proteus vulgaris (P. vulgaris), Salmonella Typhi (S. Typhi), Shigella sonnei (S. sonnei), and methicillin-resistant Staphylococcus aureus (MRSA). Both raw natural and commercial honey exhibited antibacterial properties against tested Gram-positive and Gram-negative bacteria. The tested organisms showed low sensitivity to antibiotic Etest. Conclusion. All of the bacterial species studied were uniformly receptive to all raw and commercial tested honey samples; in contrast, the tested organisms showed low sensitivity to antibiotics. Commercial honey has the same antibacterial activity as the raw natural unprocessed honey against tested clinical isolates. Thus, honey is a successful alternative to conventional antibiotics as has been proved against clinical isolates.
\end{abstract}

\section{Introduction}

Honey is a viscous sugar solution made by honey bees from plant nectar and honeydew. Honey has been used for medicinal purposes for centuries and has been reintroduced in modern medical practice. Composition of honey differs depending on the plant source, but the key components of the bee forage are identical to those found in all honeys [1].

Honey antimicrobial activity is the main factor for wound protection due to osmolarity effect, elevated concentration of sugar, low water content, and low $\mathrm{pH}[2,3]$.

Topical application of honey to the first- and seconddegree burns was found to be effective in decreasing morbidity and reducing time duration required for recovery [4].
Australian Jelly bush honey is a commercial honey which has been marked as bioactive honey. Antibacterial activity was due to other causes, such as bee-defensin-1 peptides and phenolic compounds [5].

Honey exerted bacteriostatic and bactericidal activity against methicillin-resistant $S$. aureus isolates from infected wound [6].

Some honeys demonstrate a wide range of antimicrobial activities against resistant bacteria [7].

Phenyllactic acid and methyl syringate were considered as fingerprint and chemical markers of Agastache honey and honey of Leptospermum sources [8]. Free phenols (volatile compounds), phenolic acids, polyphenols (usually in the form of flavonoids), anthocyanins, procyanidins, and pigments are 
found in the nectar honey phenolic compounds [9]. Manuka honey used in wound-care products may tolerate dilution with large amounts of wound exudate while still remaining active enough to suppress bacterial development [10].

Broad-spectrum antimicrobial activity of honey samples from Kosovo (antibacterial, antifungal, antiviral, and antimycobacterial properties) has been confirmed in in vitro and in vivo studies and was considered due to its acidity (low $\mathrm{pH}$ ), osmotic effect, high sugar levels, and presence of bacteriostatic and bactericidal factors (hydrogen peroxide, antioxidants, lysozyme, polyphenols, phosphate, phenolic acid, and flavonoids) [11].

In particular, honey demonstrated high antimicrobial activity against different strains of Staphylococcus and $S$. Typhimurium ATCC 51812 [12].

It has been shown that undiluted and diluted honey at $75,50,30$, and $10 \%$ is effective in inhibiting $S$. aureus and $S$. epidermidis [12]. Comparative research of antibacterial of honey in various dilutions to a number of commonly used antibiotics against nine pathogens from urine specimens found that honey samples tested are superior to all tested antibiotics [13]. Local honey obtained from Nigeria was tested in vitro against enteropathogen isolates; undiluted honey and different honey concentrations inhibit all the enteropathogens tested $[14,15]$.

Bacteriostatic and bactericidal properties are identified in honey, against methicillin-resistant Staphylococcus aureus (MRSA), [16]. Honey activity against Helicobacter pylori ( $H$. pylori) has been reported in vitro using agar diffusion assay [17].

Two enzymes, urease and xanthine oxidase, which are important virulence factors of $H$. Pylori, are effectively inhibited by honey phenolic components. These findings substantially confirm that regular consumption of honey (particularly polyphenol-rich foods) can help to prevent gastric ulcers due to H. pylori [18].

Different dilutions of honey samples collected from Ethiopia have both bacteriostatic and bactericidal activities against MRSA isolation from wound infection [19].

Strong antistaphylococcal honey activity has also been reported in MRSA clinical isolates (methicillin-resistant Staphylococcus aureus). Efficient inhibition growth was reported for MRSA isolates [20, 21]. Honey effectively eradicates $P$. aeruginosa-formed biofilm [22]. MRSA and S. aureus methicillinsensitive (MSSA) measured honey was found to be susceptible with an inhibition zone of $36.2 \pm 0.2 \mathrm{~mm}$ and $40.16 \pm 0.152$ $\mathrm{mm}$, respectively [23].

Three honey samples were obtained from Baghdad tested for antibacterial activity. Three different concentrations were used 100, 70, and 50\% against different organisms. The highest antibacterial activity of honey was reported on $100 \%$ concentration [24]. The activity of Danish honey was mostly considered due to hydrogen peroxide content [21].

The difference in honey's antibacterial activity depends on the floral origin of the honey [25]. Honey rich multiflora not only improves the nutritional quality and antimicrobial ability of several clinically significant microorganisms but also increases their nutritional potential [26]. Antimicrobial activity can be attributed to a variety of factors, in addition to the presence of hydrogen peroxide $\left(\mathrm{H}_{2} \mathrm{O}_{2}\right)$, osmolarity, and acidity [27].

Water-dilute honeys (Sidr and Talh) had higher antimicrobial activity against bacterial strains than broad-spectrum antibacterial antibiotics (tetracycline and chloramphenicol), but they were less effective against fungal strains than antimicrobial antibiotics (Flucoral and mcosat) [28].

The aim of this study was to evaluate the antibacterial effect of global raw and commercial bee honey samples of different floral origins against 8 clinical isolates obtained from patients at private hospital from Sudan.

\section{Materials and Methods}

2.1. Bee Honey Samples. Thirty-two various global raw bee honey samples were obtained from different apiaries, as well as commercially sold honey samples from the local market, various brands, of different floral origins, between November and December 2018. The beekeepers determined the floral source of the honey based on the availability of flora for nectar foraging, the location of the apiary, and the honey's organoleptic qualities. Honey samples were stored in a sterile glass jar at room temperature. Samples were labeled according to the source, location, $\mathrm{pH}$, date of collection, and floral origin as shown in Table 1.

2.2. Clinical Isolates. Eight clinical isolates were collected randomly from nose, throat, ear, wound, urine, stool, and conjunctival swabs, from both male and female patients at private hospital from Sudan (December, 2018), before antibiotic treatment. Sterile cotton swabs were used. The samples were immediately put in screw capped bottles containing transport medium. The collected isolates were used for conventional microbiological identification.

\subsection{In Vitro Antibacterial Activity of Bee Honey}

2.3.1. Inoculum Preparation. Pure culture and standard inoculum size was maintained for antibacterial susceptibility. Clinical isolates were obtained from patients at private hospital (Sudan); were suspended in a sterile saline to match a $0.5 \mathrm{McF}$ arland standard tube, which is accessible on the market; and provide a density of $1.5 \times 10^{8}$ colony-forming units $(\mathrm{CFU} / \mathrm{ml})$.

2.3.2. Disk Diffusion Susceptibility Test. The assay method was adapted from Allen et al. [29].

The 8 clinical isolates were identified by conventional microbiological methods and used for susceptibility test.

Mueller Hinton agar was utilized as the culture medium, reconstituted, sterilized (using autoclave) at $121^{\circ} \mathrm{C}$ for 15 minutes, left to set at $48^{\circ} \mathrm{C}$, and inoculated with $0.1 \mathrm{ml}$ of standardized 24 broth culture of bacterial suspensions that match the turbidity of the $0.5 \mathrm{McFarland}$ standard tube $\left(1.5 \times 10^{8}\right)(\mathrm{FU} / \mathrm{ml})$. The guidelines for their use, as well as recommendations for how to use them, are provided by the National Committee for Clinical Laboratory Standards (NCCLS) (940 W. Valley Road, Suite 1400, Wayne, PA, 1987). The prepared Mueller Hinton medium was distributed aseptically in $20 \mathrm{ml}$ volumes into sterile Petri dishes 
Table 1: Thirty-two different global honey samples collected from different countries.

\begin{tabular}{|c|c|c|c|}
\hline Code & Locality & Source & Floral origin $\mathrm{pH}$ \\
\hline A & Sudan & East Singa & Neem 3.4 \\
\hline B & Sudan & East Singa & Sidr 4.6 \\
\hline $\mathrm{C}$ & Sudan & East Singa & Sidr 4.9 \\
\hline $\mathrm{D}_{1}$ & Sudan & Damazine east & Sidr 4.7 \\
\hline $\mathrm{D}_{2}$ & Sudan & West apiary & Acacia 4.3 \\
\hline $\mathrm{E}$ & Sudan & West Darfur apiary & Sidr 4.5 \\
\hline $\mathrm{F}$ & Sudan & West Darfur apiary & Mountain 4.6 \\
\hline G & Sudan & South apiary & Acacia 4.2 \\
\hline $\mathrm{H}$ & Sudan & Darfur apiary & Acacia 3.5 \\
\hline $\mathrm{I}_{1}$ & Saudi Arabia & College of Agriculture apiary & Sun flower 3.5 \\
\hline $\mathrm{I}_{2}$ & Saudi Arabia & College of Agriculture apiary & Sun flower 3.5 \\
\hline $\mathrm{J}$ & Kashmir & Commercial & Sun flower 3.5 \\
\hline K & Yemen & Apiary & Sidr 3.5 \\
\hline $\mathrm{L}_{1}$ & Kashmir & Apiary & Sidr 3.5 \\
\hline $\mathrm{L}_{2}$ & India & Apiary & Unknown 3.5 \\
\hline M & Iran & Commercial & Sidr 4.2 \\
\hline $\mathrm{N}$ & Turkey & Commercial & Orange 3.5 \\
\hline $\mathrm{O}$ & Saudi Arabia & Al-Shifa commercial & Flowers 4.2 \\
\hline $\mathrm{P}$ & Australia & Commercial & Flowers 4.1 \\
\hline Q & Argentine & Commercial & Orange 4.3 \\
\hline $\mathrm{R}$ & Saudi Arabia & Al-Shifa commercial & Orange 4.0 \\
\hline S & United stated & Goody commercial & Flowers 3.8 \\
\hline $\mathrm{T}_{1}$ & Germany & Langnese commercial & Flowers 3.7 \\
\hline $\mathrm{T}_{2}$ & Germany & Langnese commercial & Flowers 3.7 \\
\hline $\mathrm{U}$ & Saudi Arabia & Al-Shifa commercial & Acacia 4.2 \\
\hline $\mathrm{V}$ & Palestinian & Apiary & Citrus 4.2 \\
\hline $\mathrm{W}_{1}$ & Egypt & Apiary & Alfa 4.6 \\
\hline $\mathrm{W}_{2}$ & Egypt & Apiary & Alfa 4.5 \\
\hline $\mathrm{X}$ & Jordan & Apiary & Citrus 4.5 \\
\hline $\mathrm{Y}_{1}$ & Yemen & Apiary & Sidr 4.4 \\
\hline $\mathrm{Y}_{2}$ & Yemen & Commercial & Sidr 4.5 \\
\hline $\mathrm{Z}^{2}$ & Saudi Arabia & Tabuk apiary & Sun flower 4.3 \\
\hline
\end{tabular}

(95 mm internal diameter) and permitted to set. Sterile swabs were dipped in the fresh overnight microorganism suspension. The excess fluid was pressed against the wall tube then swabbed over the Mueller Hinton plates. Gently pressed over the top of the swabbed plate was the six-millimeter sterile filter paper disk impregnated with the honey sample $(0.6 \mu)$. The disk diffusion plate technique has been used to examine the antibacterial activity of bee honey.

The inoculated plates were incubated for 18-24 hours at $37^{\circ} \mathrm{C}$. The diameter of the growth inhibition zone that resulted was measured in millimeter. In four replicates, each honey sample was examined against tested organism. The average diameter of the inhibition zone was measured.

\subsubsection{Epsilometer Test (Etest)}

(1) Predefined Gradient for MIC Determinations. The Etest is a quantitative tool for assessing antimicrobial resistance of various bacterial strains. The method consists of an antibiotic gradient that is used to evaluate the minimum inhibitory concentration (MIC), in micrograms per milliliter, of various antibacterial agents against different microorganisms when measured on inoculated agar media.

A plastic strip that is thin, sterile, and nonporous, of gradient antibiotic , $5 \mathrm{~mm}$ wide and $60 \mathrm{~mm}$ long, is used in the Etest. The obtained result of MIC reading scale in grams per milliliter of the strip is printed on one side, with a two-letter code on the handle showing the exact antibiotic term, with the highest concentration at the top and lowest concentration at the bottom. The gradient spans 15 twofold dilutions of the traditional MIC form, covering a continuous concentration spectrum.

2.3.4. Procedure for Etest. The following 6 Etests amoxicillin, ciprofloxacin, cefotaxime, chloramphenicol, gentamicin, and 
TABLE 2: Antibacterial activity of 32 bee honeys against 8 clinical isolates.

\begin{tabular}{|c|c|c|c|c|c|c|c|c|}
\hline \multirow{2}{*}{ Honey samples } & \multicolumn{8}{|c|}{ Bacterial strain } \\
\hline & S. aureus & E. coli & K. pneumoniae & P. aeruginosa & Proteus & S. Typhi & S. sonnei & MRSA \\
\hline & \multicolumn{8}{|c|}{ Inhibition zone in $\mathrm{mm} \pm(\mathrm{SD})$} \\
\hline A & $25 \pm(0.5)$ & $22 \pm(0.5)$ & $22 \pm(0.5)$ & $25 \pm(0.5)$ & $23 \pm(0.5)$ & $21 \pm(0.5)$ & $22 \pm(0.5)$ & $24 \pm(0.5)$ \\
\hline B & $25 \pm(0.7)$ & $20 \pm(0.5)$ & $21 \pm(0.6)$ & $24 \pm(0.6)$ & $22 \pm(0.5)$ & $22 \pm(0.5)$ & $23 \pm(0.5)$ & $25 \pm(0.6)$ \\
\hline $\mathrm{C}$ & $24 \pm(0.5)$ & $24 \pm(0.5)$ & $21 \pm(0.5)$ & $31 \pm(0.6)$ & $24 \pm(0.5)$ & $19 \pm(0.5)$ & $24 \pm(0.5)$ & $23 \pm(0.6)$ \\
\hline $\mathrm{D}_{1}$ & $22 \pm(0.5)$ & $19 \pm(0.5)$ & $20 \pm(0.5)$ & $(0.5) 38 \pm$ & $25 \pm(0.6)$ & $20 \pm(0.5)$ & $22 \pm(0.5)$ & $22 \pm(0.5)$ \\
\hline $\mathrm{D}_{2}$ & $24 \pm(0.5)$ & $20 \pm(0.5)$ & $21 \pm(0.5)$ & $27 \pm(0.4)$ & $24 \pm(0.4)$ & $25 \pm(0.5)$ & $24 \pm(0.4)$ & $21 \pm(0.5)$ \\
\hline $\mathrm{E}$ & $(0.5) 24 \pm$ & $19 \pm(0.5)$ & $20 \pm(0.5)$ & $28 \pm(0.6)$ & $23 \pm(0.5)$ & $22 \pm(0.4)$ & $23 \pm(0.6)$ & $22 \pm(0.4)$ \\
\hline $\mathrm{F}$ & $25 \pm(0.6)$ & $20 \pm(0.5)$ & $21 \pm(0.5)$ & $26 \pm(0.5)$ & $22 \pm(0.4)$ & $22 \pm(0.5)$ & $22 \pm(0.4)$ & $24 \pm(0.5)$ \\
\hline G & $23 \pm(0.5)$ & $25 \pm(0.4)$ & $22 \pm(0.5)$ & $25 \pm(0.4)$ & $23 \pm(0.5)$ & $23 \pm(0.4)$ & $21 \pm(0.4)$ & $25 \pm(0.5)$ \\
\hline $\mathrm{H}$ & $24 \pm(0.4)$ & $22 \pm(0.5)$ & $21 \pm(0.4)$ & $21 \pm(0.4)$ & $23 \pm(0.5)$ & $21 \pm(0.5)$ & $20 \pm(0.5)$ & $23 \pm(0.5)$ \\
\hline $\mathrm{I}_{1}$ & $26 \pm(0.5)$ & $23 \pm(0.6)$ & $22 \pm(0.4)$ & $23 \pm(0.6)$ & $24 \pm(0.4)$ & $22 \pm(0.4)$ & $22 \pm(0.4)$ & $24 \pm(0.6)$ \\
\hline $\mathrm{I}_{2}$ & $27 \pm(0.5)$ & $22 \pm(0.5)$ & $23 \pm(0.6)$ & $23 \pm(0.5)$ & $25 \pm(0.5)$ & $23 \pm(0.6)$ & $23 \pm(0.5)$ & $21 \pm(0.5)$ \\
\hline $\mathrm{J}$ & $23 \pm(0.6)$ & $21 \pm(0.5)$ & $22 \pm(0.5)$ & $25 \pm(0.6)$ & $26 \pm(0.5)$ & $24 \pm(0.5)$ & $25 \pm(0.5)$ & $22 \pm(0.5)$ \\
\hline $\mathrm{K}$ & $24 \pm(0.4)$ & $18 \pm(0.5)$ & $23 \pm(0.6)$ & $24 \pm(0.4)$ & $24 \pm(0.6)$ & $25 \pm(0.6)$ & $22 \pm(0.4)$ & $23 \pm(0.5)$ \\
\hline $\mathrm{L}_{1}$ & $22 \pm(0.4)$ & $17 \pm(0.4)$ & $23 \pm(0.5)$ & $24 \pm(0.4)$ & $24 \pm(0.5)$ & $23 \pm(0.4)$ & $21 \pm(0.5)$ & $24 \pm(0.4)$ \\
\hline $\mathrm{L}_{2}$ & $22 \pm(0.6)$ & $22 \pm(0.5)$ & $22 \pm(0.5)$ & $22 \pm(0.4)$ & $25 \pm(0.5)$ & $24 \pm(0.4)$ & $23 \pm(0.5)$ & $22 \pm(0.4)$ \\
\hline M & $23 \pm(0.5)$ & $22 \pm(0.5)$ & $23 \pm(0.5)$ & $20 \pm(0.4)$ & $23 \pm(0.5)$ & $22 \pm(0.4)$ & $24 \pm(0.5)$ & $21 \pm(0.4)$ \\
\hline $\mathrm{N}$ & $22 \pm(0.5)$ & $23 \pm(0.5)$ & $22 \pm(0.5)$ & $24 \pm(0.4)$ & $24 \pm(0.5)$ & $23 \pm(0.5)$ & $23 \pm(0.5)$ & $23 \pm(0.5)$ \\
\hline $\mathrm{O}$ & $21 \pm(0.4)$ & $20 \pm(0.5)$ & $23 \pm(0.5)$ & $23 \pm(0.4)$ & $24 \pm(0.5)$ & $21 \pm(0.4)$ & $22 \pm(0.5)$ & $25 \pm(0.4)$ \\
\hline $\mathrm{P}$ & $23 \pm(0.5)$ & $21 \pm(0.5)$ & $23 \pm(0.5)$ & $23 \pm(0.5)$ & $23 \pm(0.4)$ & $22 \pm(0.4)$ & $21 \pm(0.4)$ & $24 \pm(0.4)$ \\
\hline Q & $24 \pm(0.5)$ & $20 \pm(0.5)$ & $21 \pm(0.6)$ & $23 \pm(0.5)$ & $25 \pm(0.5)$ & $23 \pm(0.6)$ & $22 \pm(0.5)$ & $23 \pm(0.5)$ \\
\hline $\mathrm{R}$ & $23 \pm(0.6)$ & $21 \pm(0.5)$ & $22 \pm(0.4)$ & $22 \pm(0.5)$ & $22 \pm(0.5)$ & $23 \pm(0.4)$ & $23 \pm(0.4)$ & $22 \pm(0.5)$ \\
\hline S & $24 \pm(0.5)$ & $22 \pm(0.5)$ & $24 \pm(0.4)$ & $23 \pm(0.5)$ & $23 \pm(0.6)$ & $22 \pm(0.5)$ & $24 \pm(0.6)$ & $21 \pm(0.4)$ \\
\hline $\mathrm{T}_{1}$ & $25 \pm(0.4)$ & $20 \pm(0.5)$ & $22 \pm(0.6)$ & $22 \pm(0.5)$ & $24 \pm(0.5)$ & $24 \pm(0.5)$ & $23 \pm(0.5)$ & $24 \pm(0.5)$ \\
\hline $\mathrm{T}_{2}$ & $24 \pm(0.6)$ & $21 \pm(0.5)$ & $21 \pm(0.4)$ & $25 \pm(0.5)$ & $25 \pm(0.4)$ & $23 \pm(0.5)$ & $22 \pm(0.5)$ & $24 \pm(0.6)$ \\
\hline $\mathrm{U}$ & $25 \pm(0.5)$ & $22 \pm(0.6)$ & $21 \pm(0.5)$ & $24 \pm(0.5)$ & $24 \pm(0.5)$ & $22 \pm(0.5)$ & $23 \pm(0.4)$ & $25 \pm(0.5)$ \\
\hline V & $23 \pm(0.5)$ & $21 \pm(0.4)$ & $22 \pm(0.5)$ & $25 \pm(0.4)$ & $22 \pm(0.5)$ & $24 \pm(0.6)$ & $22 \pm(0.5)$ & $23 \pm(0.6)$ \\
\hline $\mathrm{W}_{1}$ & $22 \pm(0.5)$ & $22 \pm(0.5)$ & $20 \pm(0.5)$ & $24 \pm(0.5)$ & $21 \pm(0.5)$ & $22 \pm(0.4)$ & $21 \pm(0.5)$ & $22 \pm(0.5)$ \\
\hline $\mathrm{W}_{2}$ & $21 \pm(0.5)$ & $20 \pm(0.6)$ & $21 \pm(0.5)$ & $23 \pm(0.6)$ & $23 \pm(0.5)$ & $21 \pm(0.5)$ & $22 \pm(0.5)$ & $21 \pm(0.4)$ \\
\hline $\mathrm{X}$ & $24 \pm(0.6)$ & $22 \pm(0.4)$ & $22 \pm(0.5)$ & $22 \pm(0.5)$ & $24 \pm(0.4)$ & $22 \pm(0.5)$ & $23 \pm(0.5)$ & $22 \pm(0.6)$ \\
\hline $\mathrm{Y}_{1}$ & $23 \pm(0.5)$ & $21 \pm(0.5)$ & $21 \pm(0.5)$ & $23 \pm(0.5)$ & $22 \pm(0.5)$ & $22 \pm(0.6)$ & $24 \pm(0.5)$ & $23 \pm(0.5)$ \\
\hline $\mathrm{Y}_{2}$ & $22 \pm(0.4)$ & $20 \pm(0.5)$ & $21 \pm(0.4)$ & $22 \pm(0.6)$ & $21 \pm(0.5)$ & $21 \pm(0.5)$ & $23 \pm(0.5)$ & $21 \pm(0.4)$ \\
\hline $\mathrm{Z}$ & $23 \pm(0.5)$ & $22 \pm(0.4)$ & $21 \pm(0.4)$ & $22 \pm(0.5)$ & $21 \pm(0.5)$ & $23 \pm(0.5)$ & $22 \pm(0.4)$ & $21 \pm(0.5)$ \\
\hline
\end{tabular}

tetracycline were used against 8 clinical isolates: S. aureus, E. coli, Klebsiella spp., Proteus vulgaris., Salmonella spp., Shigella spp., Pseudomonas spp., and methicillin-resistant $S$. aureus (MRSA).

Suspension of bacteria equivalent to a $0.5 \mathrm{McFarland}$ tube was used to swab the surface of Mueller Hinton agar plate evenly, by sterile forceps. A strip of the Etest was removed and pressed on the inoculated plate; the whole strip is in total contact with the surface of the agar. For single
MIC, two strips were used on a $90 \mathrm{~mm}$ agar plate. The inoculated medium was incubated at $37 \mathrm{C}^{\circ}$ for 24 hours.

\section{Results}

Identification of the 8 clinical isolates obtained from patients at private hospital (Sudan) was carried out by the conventional microbiological methods at the Microbiology Laboratory. The following bacterial strains were identified as 
Table 3: Minimum inhibitory concentration of 6 antibiotics against 8 clinical isolates (Etest).

\begin{tabular}{lcccccccc}
\hline \multirow{2}{*}{ Antibiotics } & E. coli & Klebsiella & Proteus vulgaris & Salmonella & Shigella & Pseudomonas & MSSA & MRSA \\
\hline \multicolumn{7}{c}{} & \multicolumn{7}{c}{ MIC (mg/l) } \\
\hline Amoxicillin & 3 & 3 & 0.125 & 1.5 & 0.6 & 3 & 4 & 0.125 \\
Ciprofloxacin & 2 & 0.125 & 0.5 & 2.5 & 0.18 & 0.125 & 3 & 0.50 \\
Cefotaxime & 0.5 & 0.6 & 0.125 & 0.6 & 0.125 & 0.125 & 3 & 1.0 \\
Chloramphenicol & 1.5 & 3 & 0.50 & 3 & 2 & 1.5 & 0.125 & 3 \\
Gentamicin & 3 & 1.5 & 0.50 & 0.125 & 1.5 & 0.125 & 3 & 3 \\
Tetracycline & 4 & 1.5 & 0.064 & 0.125 & 0.50 & 0.50 & 0.125 & 1.0 \\
\hline
\end{tabular}

Staphylococcus aureus, methicillin-resistant Staphylococcus aureus (MRSA), E. coli, K. pneumoniae, $P$. aeruginosa, $P$. vulgaris, $S$. Typhi, and S. sonnei.

In vitro antibacterial effects against 8 clinical isolates, $S$. aureus, E.coli, $K$. pneumoniae, $P$. aeruginosa, $P$. vulgaris, $S$. Typhi, S. sonnei, and methicillin-resistant S. aureus (MRSA), were carried out by using 32 raw natural and commercial honeys. Both Gram-positive and Gram-negative bacteria were inhibited by 32 tested honey samples (Table 2).

Commercial honey samples exhibited antibacterial activity against all the 8 clinical isolates (Table 2).

In the present study, Gram-negative bacteria $P$. aeruginosa demonstrated the highest susceptibility towards different honeys tested. Sample $\mathrm{D}_{1}$ obtained from Damazine east of Sudan floral origin-sidr-was found to be the most active sample against $P$. aeruginosa (Table 2).

Antibacterial activity was observed in both natural and commercial honey samples against Salmonella Typhi (Table 2). Sample $\mathrm{L}_{1}$ obtained from Kashmir, floral origin sidr; sample $\mathrm{L}_{2}$ obtained from India; sample $M$ from Iran commercial source; and sample X Jordanian allocation were revealed to be extremely effective against Salmonella Typhi, while samples $\mathrm{C}$ and $\mathrm{D}_{1}$ obtained from Sudan showed the least activity (Table 2).

P. vulgaris, Klebsiella spp., and Shigella spp. showed a consistent level of susceptibility to all honey tested. Samples $\mathrm{D}_{1}, \mathrm{I}_{2}$, $\mathrm{U}$, and $\mathrm{J}$ showed the highest activity against Proteus vulgaris (Table 2). MRSA was subjected to antibacterial activity using the 32 honey samples and showed similar sensitivity to all honeys tested. Samples $G, A, V, D_{1}$, and $U$ exhibited the highest activity (Table 2). The tested antibiotics against different organisms showed low MIC (Table 3). On the other hand, most of the clinical isolates were consistently found susceptible to all honeys tested.

\section{Discussion}

Controlling the rise in antibiotic resistance is one of the most pressing issues facing modern health care. Resistance to frequently used community antimicrobials is rising, according to international resistance surveillance studies. Honey has been valued for its medicinal properties since ancient times. The topical application of honey, as wound dressing, has got a lot of attention in recent years. Honey has been applied successfully to treat infections that have stopped responding to traditional antiseptic and antibiotic care. Honey has different types and levels of antibacterial activity depending on the floral source. The obtained previous favorable results previous of antibacterial activity of honey against standard organisms, it is encouraged to explore the activity of honey against clinical isolates [30].

In vitro antibacterial activity of honey against 8 clinical isolates showed different susceptibility patterns on both Gram-positive and Gram-negative bacteria. S. aureus was found to be the most sensitive to different honey samples. The present findings confirmed that the 12 commercial honey samples with different brands exhibited antibacterial activity against the 8 clinical isolates (Table 2). This proved that commercial honey has the same activity as the raw natural unprocessed honey. Previous studies documented that Australian Jelly bush honey is a commercial honey that has been marked as bioactive honey [5].

The actual impact of bee honey's geographical origin on its antibacterial activity was more pronounced in the case of S. aureus as sample $\mathrm{I}_{2}$ obtained from Saudi Arabia floral origin. Sunflower was found to be more effective against different tested organisms (Table 2). The content of phenolic acid in honey is affected by geographical location and the source of nectar plants [3]. These data imply that the connection between antibacterial activity, floral source, and environmental variables varies by geographical area [3].

Our results revealed that both natural and commercial honeys showed identical sensitivity towards the most tested microorganisms. Antibacterial activity against $S$. Typhi, $P$. vulgaris, Klebsiella, and Shigella exhibited similar sensitivity to all honeys tested. These results seems in agreement with the previous findings [5]. S. Typhi was noted to be sensitive to the most of honey tested $[12,30]$. Bacterial adhesion to epithelial cells in the mucosa is thought to be the first stage in the enhancement of a bacterial infection of the gastrointestinal tract. Since disrupting pathogenic microorganisms' attachment to the intestinal epithelium is a possible technique for controlling disease, honey is useful in the treatment of enteric pathogens, as well as inhibiting their growth and reducing their attachment to the epithelial cells of the gastrointestinal tract [28].

MRSA was subjected to antibacterial activity using the 32 honey samples and showed similar sensitivity to all honeys 
tested. Sample B, G, V, and P exhibited the highest activity against MRSA. These are in line with the previous findings which confirmed the effectiveness of different honey samples against MRSA $[6,17,20,21]$. MRSA has been a frequent cause of hospital-acquired infection and a major cause of serious infection, as well as had a pattern of resistance, not only to methicillin but also to aminoglycoside and cephalosporin [29].

The geographical and floral origin of honey samples was more pronounced on the result obtained with sample $\mathrm{D}_{2}$ Damazine east of Sudan, floral origin; sidr against Pseudomonas demonstrated the highest activity. The influnence of geographical source of bee hone on its antibacterial activity wasmore pronounced against Pseudomonas with sample D2-east of Sudan floral orgin sidr. Honey effectively eradicates $P$. aeruginosa-formed biofilm [22]. Pseudomonas spp. were well known, as a multi-drug-resistant organism. It was previously thought to be the most common cause of hospital-acquired infection. This wide range of honey sample inhibitory effects suggests differences in antimicrobial constituent (s) of honey. Similar previous findings referred the antimicrobial property of honey to different constituents [11]. Significant antibacterial activity of different floral honeys was proved against a wide range of Gram-positive and Gram-negative bacterial standard organisms [30]. The promising effects of our previous investigation on honey's antibacterial activity against standard organisms encourged to examine honey's antibacterial activity against clinical isolates.

The clinical isolates were tested for their in vitro sensitivity test against the 8 antibiotics. Bacterial strains showed low-sensitivity MIC towards antibiotics tested, as compared with honey antibacterial activity (Table 3).

The current study highlights the susceptibility of Pseudomonas spp. to the most honey samples (raw and commercial). Pseudomonas spp. among the Gram-negative bacteria revealed resistance to the majority of antibiotics tested [30]. P. aeruginosa was a common infection seen in hospitals. Honey was reported to be more effective than antibiotics at controlling bacterial growth. In the present research, raw and commercial honey has been found to be a potent antimicrobial natural product against a variety of microorganisms, including multiresistant strains. These observations were concomitant with the findings of the previous studies $[22,23,28]$. Honey has been used effectively to treat chronic infections that have failed to react to standard antiseptics and antibiotics. These criteria show promise in the treatment of bacterial infections, especially in the clinical treatment of ulcers, bed sores, burns, fractures, and surgical wounds. Honey's antimicrobial properties may be especially beneficial against bacteria that have developed antibiotic resistance. Broad-spectrum antimicrobial activity of honey can be attributed to a variety of factors, in addition to the presence of hydrogen peroxide $\left(\mathrm{H}_{2} \mathrm{O}_{2}\right)$, osmolarity, and acidity [27]. Our results revealed that the antibacterial activity of different honey samples could explain the floral diversity according to the geographic location. A previous study confirmed that difference in honey's antibacterial activity depends on the floral origin of the honey [25]. The current study highlights that tested antibiotics showed selective inhibition of the clinical isolates, which was not the case with the honey samples. Honey superiority over antibiotics over a wide variety of microbes was reported. These results are in accordance with a previous comparative study of honey to a number of commonly used antibiotics against 9 pathogens isolated from urine specimens and showed that tested honey samples is superior to all antibiotics [13].

Honey has also been reported to be more effective as an antibacterial agent against several Pseudomonas and Staphylococcus strains than antibiotic gentamicin [28]. To demonstrate the synergistic action of honey and antibiotics, honey can be given orally with antibiotics [10]. The inhibitory impact of various floral honey samples against resistant microbes that taint wounds and hinder wound healing would pave the way for honey to be reintroduced into modern medicine. The limitation of honey's medical usefulness in vivo is being studied for future medical practice. To produce the active component as a pharmaceutical product, more research on the active compounds of efficient antibacterial action of natural bee honey is required. Hence, there is a need to find natural alternative treatment to counter the multi-drug-resistant organisms. A traditional therapy therefore appears to have enormous potential in solving new clinical problems. To assess the potency of raw natural and commercial honey against isolated clinical isolates, further investigations should be conducted against antibioticresistant microorganisms.

\section{Conclusion}

Both natural and commercial honey samples exerted inhibitory effects on the various Gram-positive and Gram-negative bacteria understudy. Commercial honey has the same activity as the raw natural unprocessed honey. Honey was found to be effective in inhibiting $P$. aeruginosa and MRSA. Thus, honey is highly recommended as wound dressing to manage wound healing due to its antibacterial activity against a wide range of microorganisms. Geographical and botanical sources were more pronounced on honey antibacterial properties. Antibacterial activity in honey is influenced by a variety of factors that are not entirely dependent on the floral source. Honey could be an alternative treatment approach in chronic wounds and burns of different natures in inhibiting different types of microorganisms not responding to conventional antibiotics without side effects. Further research efforts would be beneficial in attempt to control resistant organisms not responding to antibiotic treatment. Using honey in a medical setting may reduces financial cost and hospital stay.

\section{Data Availability}

The datasets used and analyzed in the current study are included in the article.

\section{Ethical Approval}

Ethical approval was obtained from the ethical committee at the private hospital at Sudan to get the clinical isolates collected already for laboratory diagnosis. 


\section{Disclosure}

The manuscript has been uploaded as preprint by Research Square.

\section{Conflicts of Interest}

The author declared that there is no conflict of interest.

\section{Acknowledgments}

This research was funded by the Deanship of Scientific Research at Princess Nourah Bint Abdulrahman University through the Fast-track Research Funding Program.

\section{References}

[1] R. J. Weston, L. K. Brocklebank, and Y. Lu, "Identification and quantitative levels of antibacterial components of some New Zealand honeys," Food Chemistry, vol. 70, no. 4, pp. 427435, 2000.

[2] Y.-T. Khoo, A. S. Halim, K.-K. B.-K. Singh, and N.A. Mohamad, "Wound contraction effects and antibacterial properties of Tualang honey on full-thickness burn wounds in rats in comparison to hydrofibre," BMC Complementary and Alternative Medicine, vol. 10, no. 1, 2010.

[3] H. A. L. Wahdan, "Causes of the antimicrobial activity of honey," Infection, vol. 26, no. 1, pp. 26-31, 1998.

[4] P. S. Baghel, S. Shukla, R. K. Mathur, and R. Randa, "A comparative study to evaluate the effect of honey dressing and silver sulfadiazene dressing on wound healing in burn patients," Indian Journal of Plastic Surgery, vol. 42, no. 2, 2009.

[5] P. H. Kwakman and S. A. Zaat, "Antibacterial components of honey," IUBMB Life, vol. 64, no. 1, pp. 48-55, 2012.

[6] M. Mama, T. Teshome, and J. Detamo, "Antibacterial activity of honey against methicillin-resistant Staphylococcus aureus: a laboratory-based experimental study," International Journal of Microbiology, vol. 2019, Article ID 7686130, 9 pages, 2019.

[7] R. Wang, M. Starkey, R. Hazan, and L. G. Rahme, "Honey's ability to counter bacterial infections arises from both bactericidal compounds and QS inhibition," Frontiers in Microbiology, vol. 3, p. 144, 2012.

[8] S. Anand, M. Deighton, G. Livanos, P. D. Morrison, E. C. K. Pang, and N. Mantri, "Antimicrobial activity of $<\mathrm{i}>$ Agastache $</ \mathrm{i}>$ honey and characterization of its bioactive compounds in comparison with important commercial honeys," Frontiers in Microbiology, vol. 10, 2019.

[9] M. C. Carson, "Ion-pair solid-phase extraction," Journal of Chromatography. A, vol. 885, pp. 343-350, 2000.

[10] P. Molan and T. Rhodes, "Honey: A biologic wound dressing," Wounds, vol. 27, no. 6, pp. 141-151, 2015.

[11] B. S. Tülay and A. Hanife, "In vitro screening of antibacterial activity of honey samples collected from Kosovo," Journal of Scientific Perspectives, vol. 1, p. 2, 2017.

[12] C. Basualdo, V. Sgroy, M. S. Finola, and J. M. Marioli, "Comparisons of the antibacterial activity of honey from different provenance against bacteria usually isolated from skin wounds," Veterinary Microbiology, vol. 124, no. 3-4, pp. 375$381,2007$.

[13] A. S. Ibrahim, "Antibacterial action of honey," Bulletin of Islamic Medicine, vol. 1, pp. 363-365, 1981.
[14] C. L. Obi, E. O. Ugoji, S. A. Edun, S. F. Lawal, and C. E. Anyiwo, "The antibacterial effect of honey on diarrhea causing bacterial agentsisolated in Lagos," African Journal of Medicine and Medical Sciences, vol. 23, no. 3, pp. 257260, 1994.

[15] B. C. Nzeako and F. Al-Namaani, "The antibacterial activity of honey on Helicobacter pylori," Sultan Qaboos University Medical Journal, vol. 6, no. 2, pp. 71-76, 2006.

[16] H. Sahin, "Honey as an apitherapic product: its inhibitory effect on urease and xanthine oxidase," Journal of Enzyme Inhibition and Medicinal Chemistry, vol. 31, no. 3, pp. 1-5, 2016.

[17] O. Sherlock, A. Dolan, R. Athman et al., "Comparison of the antimicrobial activity of Ulmo honey from Chile and Manuka honey against methicillin-resistant Staphylococcus aureus, Escherichia coli and Pseudomonas aeruginosa," BMC Complementary and Alternative Medicine, vol. 10, no. 1, p. 47, 2010.

[18] W. J. Ng and M. S. Lim, "Antistaphylococcal activity of melaleuca honey," The Southeast Asian Journal of Tropical Medicine and Public Health, vol. 46, no. 3, pp. 472-479, 2015.

[19] R. Cooper, L. Jenkins, and S. Hooper, "Inhibition of biofilms of Pseudomonas aeruginosa by Medihoney in vitro," Journal of Wound Care, vol. 23, no. 3, pp. 93-104, 2014.

[20] G. N. Rani, R. Budumuru, and N. Bandaru, "Antimicrobial activity of honey with special reference to methicillin resistant Staphylococcus aureus (MRSA) and methicillin sensitive Staphylococcus aureus (MSSA)," Journal of Clinical and Diagnostic Research, vol. 11, no. 8, pp. DC05-DC08, 2017.

[21] R. D. Matzen, L. Zinck, J. Espensen et al., "The antibacterial effect in vitro of honey derived from various Danish flora," Dermatology Research and Practice, vol. 2018, Article ID 7021713, 2018.

[22] V. León-Ruiz, A. V. González-Porto, N. Al-Habsi, S. Vera, M. P. San Andrés, and P. Jauregi, “Antioxidant, antibacterial and ACE -inhibitory activity of four monofloral honeys in relation to their chemical composition," Food \& Function, vol. 4, pp. 1617-1624, 2013.

[23] M. Cenet, A. Bozdogan, G. Sezer, L. Acar, and Z. Ulukanli, "Antimicrobial activities, pollen diversity and physicochemical properties of natural honey from Southeastern Anatolia of Turkey," Advancements in Life Sciences, vol. 4, no. 2, pp. 4754, 2017.

[24] H. M. Al-Hasani and M. Hussein, "Study antibacterial activity of honey against some common species of pathogenic bacteria," Iraqi Journal of Science, vol. 59, no. 1A, pp. 3037, 2018.

[25] K. Brudzynski, K. Abubaker, M. Laurent, and A. Castle, "Reexamining the role of hydrogen peroxide in bacteriostatic and bactericidal activities of honey," Frontiers in Microbiology, vol. 2, 2011.

[26] B. M. Aminu and M. L. Khadija, "Antibacterial activity of local honey against carbapenem resistant enterobacteriaceae isolated from infected wounds," Bayero Journal of Pure \& Applied Sciences, vol. 12, pp. 13-17, 2020.

[27] A. A. Owayss, K. Elbanna, J. Iqbal et al., "In vitro antimicrobial activities of Saudi honeys originating fromZiziphus spinachristiL. andAcacia gerrardiiBenth. trees," Food Science of Nutrition, vol. 8, no. 1, pp. 390-401, 2020.

[28] Center for Disease Control and Preventions, National Center for Emerging and Zoonotic Infectious Diseases (NCEZIN), "Division of Health Care Quality Promotion (DHQP)". 
[29] K. L. Allen, P. C. Molan, and G. M. Reid, "A survey of the antibacterial activity of some New Zealand honeys," The Journal of Pharmacy and Pharmacology, vol. 43, no. 12, pp. 817-822, 1991.

[30] M. A. Wadi, "Antibacterial activity of different global honey samples against standard organisms," Asian Journal of Microbiology, Biotechnology \& Environmental Sciences, vol. 21, no. 4, pp. 924-930, 2019. 